\title{
LA MEMORIA ALLA BAROCCA NE L'ISOLA DEL GIORNO PRIMA DI UMBERTO ECO
}

\author{
Agnieszka PIWOWARSKA \\ Università Adam Mickiewicz di Poznań
}

\begin{abstract}
En): In his third novel The Island of the Day Before (L'isola del giorno prima) Umberto Eco narrates a story of an unusual castaway named Roberto who - instead of being on a desert island - finishes up on an abandoned (but only at first sight) ship. The article aims to analyze how the book's main character, during his loneliness, reminisces about events from his past. And because the novel is set in the Baroque, Roberto's memories respect the spirit of that time : numerous flashbacks are evoked by unexpected metaphors that link very distant things. The ship, which is for a young man a cumbersome prison, becomes a real Theatre of Memory, according to Giulio Camillo's idea.
\end{abstract}

Keywords (En): Umberto Eco ; The Island of the Day Before ; Memory ; Past ; Metaphor ; Baroque language

L'isola del giorno prima (1994) è ambientato nel Seicento barocco, "presumibilmente" tra il luglio e l'agosto del 1643. Il romanzo narra le avventure e le disavventure di Roberto de la Grive, nobile del Monferrato, che viene condotto dal padre all'assedio di Casale e che poi si ritrova nella Parigi di Mazzarino. Dopo vari problemi viene inviato come spia a bordo di una nave per indagare sulla questione del punto fijo, il segreto relativo al calcolo dei meridiani. La nave però affonda e Roberto, salvatosi perché legato a una tavola, naufraga su un'altra nave, la Daphne, che prima gli era parsa deserta. Essa è ancorata a poca distanza da un'isola di fronte alla quale passa - secondo i calcoli di padre Caspar, un vecchio gesuita - il meridiano antipode.

Come scrive Franco Forchetti, la memoria dei personaggi dei romanzi di Umberto Eco rappresenta il presupposto delle narrazioni (FORCHETTI, 2005: 291). $\mathrm{Ne}$ L'isola del giorno prima Roberto racconta le sue avventure tramite alcuni flashback, però in questo caso egli non è narratore: la narrazione è in terza persona e finge di basarsi sul diario del naufrago.

\section{L'idea del Teatro della Memoria. Il gusto della metafora}

Nel suo terzo romanzo, L'isola del giorno prima, Umberto Eco rivisita il topos che da molto tempo è presente nella letteratura mondiale: il topos del naufrago. ${ }^{2}$

\footnotetext{
${ }^{1}$ In merito alle citazioni tratte dal romanzo di Umberto Eco verrà utilizzata la sigla di abbreviazione IP relativa all'edizione: L'isola del giorno prima, Milano, Bompiani 2005.

2 "Il naufragio abita da secoli l'immaginario della letteratura di mare, finendo con il divenire metafora polivalente. Non possiamo raccontare la lunga avventura dell'immagine letteraria del naufragio, considerata la vastità dei riferimenti. Purtuttavia non si possono non menzionare alcuni antecedenti libreschi che, proprio perché patrimonio imprescindibile del lettore occidentale, rappresentano l'orizzonte intertestuale che si dispiega sia nell'atto di creazione del romanzo sia in quello della lettura. [...] Nella memoria letteraria affiorano il naufragio di Robinson Crusoe narrato da Defoe, i naufragi fantastici di Gulliver, i naufragi tragicomici del Candide di Voltaire, il naufragio spinoziano del Robinson di Michel Tournier, le avventure dell'Isola misteriosa di Verne e molti altri echi. La sua veneranda tradizione ne ha fatto un vero e proprio archetipo [...]." (FORCHETTI, 2005 : 119-120)
} 
Abbiamo qui a che fare però con un naufrago insolito, perché la sua sede non è un'isola deserta, ma una nave deserta. Lo stesso protagonista principale del romanzo, Roberto, è cosciente di essere un'eccezione:

"Eppure m'inorgoglisco della mia umiliazione, e poiché a tal privilegio son condannato, quasi godo di un'aborrita salvezza: sono, credo, a memoria d'uomo, l'unico essere della nostra specie ad aver fatto naufragio su di un nave deserta." (IP: 5)

Roberto si salva dal naufragio approdando su una nave deserta, la Daphne, che diventa, in un breve tempo, un piccolo labirinto, un luogo di misteri, il centro dell'assedio oceanico. F. Forchetti rileva il fatto che la Daphne per Roberto rimane un mistero, un labirinto, così come la biblioteca de Il nome della rosa. Al pari di questa, è un labirinto manierista, strutturato con tanto di intruso e sorprese meccaniche (FORCHETTI, 2005: 122). Però la nave non è solo un labirinto: è un labirinto che diventa per lui un Teatro della Memoria. Come si legge in una descrizione delle riflessioni di Roberto ne L'isola del giorno prima:

"Erano giorni che pensava al proprio passato fissando gli occhi sull'unica scena che avesse davanti, quella della Daphne, e la Daphne si stava trasformando in un Teatro della Memoria, come se ne concepivano ai suoi tempi, dove ogni tratto gli ricordava un episodio antico o recente della sua storia [...].” (IP: 100)

L'idea del Teatro della Memoria, che viene qui rievocata, fu avviata nel XVI secolo da Giulio Camillo - letterato, filosofo, mago, cabalista - che era ossessionato dal progetto di costruire un universale teatro della memoria. Viglio, testimone oculare della costruzione camilliana, la descrive dicendo che lo stesso Camillo chiama questo suo teatro con molti nomi: a volte lo chiama "mente e anima artificiale", alle volte invece "anima provvista di finestre". Secondo Viglio, egli pretende che tutte le cose che la mente umana può concepire e che non si possono vedere ad occhio nudo possano tuttavia, dopo essere state raccolte con attenta meditazione, essere espresse mediante certi simboli corporei in modo tale che l'osservatore può, all'istante, percepire con l'occhio tutto ciò che altrimenti è celato nelle profondità della mente umana. E appunto a causa di questa percezione corporea lo definisce "teatro" (YATES, 1977: 139).

Il Teatro di Giulio Camillo costituisce la possibilità di rappresentare una mappa dei nessi metafisici che fondano la realtà, attraverso "immagini capaci di svolgere un'azione, di colpire; immagini che condensano in sé emozioni e conoscenze, in un intreccio tale che l'impatto delle prime faccia scattare la catena delle seconde" (BOLZONI, 1991: 12). Queste immagini si trovano dentro i cassetti della memoria. Come scrive Marco M. Sambo:

"[...] la memoria riporta lo sguardo al passato, alla ricerca di un rinnovato sapere, un cammino verso la conoscenza da attuare mediante il Teatro Totale. E tale teatro diviene il supremo labirintico circolare dove richiamare il sapere aprendo i cassetti”.

\section{E prosegue scrivendo:}

"L'edificio ideale di Camillo rappresenta [...] il tempio di una classicità dell'idea inconscia di memoria. Il Global Theatre costituisce tanto un tempio quanto il tempo del ricordo perché i cassetti si aprono, uno alla volta. Un cassetto richiama l'altro, un altro cassetto ne richiama un 
altro ancora e la memoria si costruisce pian piano nel suo aspetto totalizzante." (SAMBO, 2004: 147-148)

Nel "teatro" di Giulio Camillo (che infatti aveva la struttura di un anfiteatro) viene raccolto ed ordinato - secondo le tecniche dell'arte della memoria - l'intero sapere umano, rispecchiando nella propria struttura quella dell'universo. Vi si intrecciano due idee: quella dell'enciclopedismo (accumulare il sapere grazie a un'organizzazione che permetta di incasellare tutta la sapienza umana) e quella del mondo-teatro. Però ciò che è più importante - e l'idea camilliana ne dà la testimonianza - è il fatto che l'arte della memoria abbia insegnato a modellare la propria mente, a dividerla in spazi ordinati, a costruire elaborate architetture interiori (BOLZONI, 1991: 12).

Roberto, assediato dall'oceano, è nel ventre di una nave che somiglia ad un teatro mnemonico che lo ossessiona con suggestioni e somiglianze (FORCHETTI, 2005: 316). Questo è naturale: già Quintiliano sapeva che i luoghi assistono la memoria, che il luogo provoca associazioni mnemoniche (YATES, 1977: 36). Ci vuole sempre qualche punto di partenza per iniziare il processo del ricordo; i nostri pensieri compiono delle operazioni sulle immagini depositate che provengono dalle percezioni sensoriali (YATES, 1977: 46). E la memoria di Roberto è un ricordare in barocco che nasce dalle sollecitazioni sensoriali e dal gusto della metafora. Il piacere della similitudine lo induce a considerare la Daphne come un teatro della memoria, dove le "vele raccolte" sono l'immagine di "lei perduta" e la galleria, da cui esplora l'isola lontana, gli evoca "la lontananza di lei" (FORCHETTI, 2005: 316).

Roberto, prima del suo viaggio, aveva avuto un contatto con uno strumento molto interessante, cioè il cannocchiale di padre Emanuele. Padre Emanuele, come sottolinea Peter Bondanella, è stato sicuramente modellato sulla personalità di uno dei più conosciuti teorici della letteratura, Emanuele Tesauro, il cui trattato sulla metafora e sul concetto era intitolato Il cannocchiale aristotelico (1665). La metafora è assolutamente indispensabile nella ricerca delle somiglianze, delle analogie e delle corrispondenze tra le cose (BONDANELLA, 1997: 176). Lo stesso Eco dice che ne Il cannocchiale Emanuele Tesauro propone un modello di metafora come modo di scoprire relazioni ancora sconosciute tra le cose. La metafora secondo lui doveva costituire lo strumento di rinnovamento delle scienze oggi chiamate umanistiche, dato che l'ingegno acuto ha la capacità di seguire una pista tra le analogie e le somiglianze. Per Tesauro trovare la metafora significa, alla Aristotele, conoscere nuove descrizioni delle cose (ECO, 2009: 36-38).

Non senza ragione Emanuele Tesauro fa riferimento ad Aristotele. La teoria aristotelica della metafora non solo costituisce il primo studio preciso su questa figura, ma inoltre non considera la metafora come una semplice decorazione di una dichiarazione, ma le attribuisce una funzione cognitiva. Lo spunto più importante incluso nella Poetica dice che la metafora è la migliore figura retorica, perché capire le metafore significa "intravedere le somiglianze". Aristotele usa il verbo theorein che significa intravedere, indagare, paragonare, giudicare. Questo interessante concetto poetico ci ordina di indagare la somiglianza che è stata suggerita, ma non è affatto evidente. Aristotele dice che bisogna trarre le metafore dalle cose non del tutto evidenti perché, come nella filosofia, l'intelletto perspicace 
riconosce, trova, intravede (theorein) le somiglianze tra cose anche molto lontane, d'altra parte afferma che le metafore contengono in sé un indovinello. Per di più, Aristotele respinge la metafora evidente, che non produce nessuna impressione: piacevole è solo ciò che desta meraviglia. Secondo lui la metafora non costituisce l'oggetto dell'imitazione ma appartiene alla sfera dell'invenzione (ECO, 2009: 5758).

Nella Retorica invece Aristotele ha insistito sul potere cognitivo della metafora, il che si manifesta sia nel fatto che la metafora ci mette sotto gli occhi qualche cosa di nuovo, lavorando su una lingua preesistente, sia nel fatto che essa ci invita a scoprire le regole di una lingua a venire. E come nota Umberto Eco, la teoria aristotelica ci pone di fronte al problema fondamentale di ogni filosofia del linguaggio, e cioè se la metafora sia uno scarto rispetto a una letterarietà soggiacente o il luogo di nascita di ogni grado zero della scrittura (ECO, 2008b: 269).

Su quei fatti è basato il concetto di metafora presente ne L'isola del giorno prima. Padre Emanuele percepiva così il ruolo della metafora:

“[...] E questo è ufficio della Figura eccelsa fra tutte, la Metafora. Se l'Ingegno, e quindi il Sapere, consistono nel legare insieme Notioni remote e trovare Simiglianza in cose dissimili, la Metafora, tra le Figure la più acuta e peregrina, è la sola capace di produrre Maraviglia, da cui nasce il Diletto, come dai cambiamenti delle scene a teatro. E se il Diletto che ci arrecano le Figure è quello d'imparar cose nuove senza fatica e molte cose in picciolo volume, ecco che la Metafora, portando a volo la nostra mente da un Genere all'altro, ci fa travedere in una sola Parola più di un Obietto." (IP: 85-86)

La Metafora Ingegnosa deve essere molto complessa. Padre Emanuele si aiuta con il suo Cannocchiale, che pare a Roberto un'immagine dell'ingegno che non intende né colpire né sedurre, bensì scoprire e rivelare connessioni tra le cose, e dunque farsi "nuovo strumento di verità". Quindi il pensare metaforicamente produce nuove verità.

Il fascino barocco delle somiglianze si manifesta all'interno del libro nel produrre metafore inaspettate. E quelle metafore, come viene descritto sotto, hanno un ruolo molto importante: sono pretesto per i ricordi del protagonista.

\section{La nave-labirinto: ricordare in barocco}

Roberto, essendo naufrago su una nave deserta, comincia a scrivere un diario, e così inizia a viaggiare à rebours nella memoria. Lo stesso Umberto Eco nel suo saggio Come scrivo spiega che cosa determina le strutture temporali dell'intreccio:

"Roberto non doveva lasciare la nave (se non alla fine, ma per fini e con esiti incerti). Dunque tutto quello che si poteva raccontare, che non avvenisse sulla nave, doveva avvenire per via di rammemorazione, a meno di appiattire l'intreccio sulla fabula e raccontare per filo e per segno di come un giovane, andato a Parigi dopo le sue avventure a Casale, si trovasse su una nave eccetera eccetera. [...] Roberto ricorda qualcosa, e intanto qualcosa accade sulla nave. Qualcosa accade sulla nave, e Roberto ricorda qualcosa. A mano a mano che i ricordi di Roberto passano dal 1630 al 1642, sulla nave gli eventi procedono ora per ora." (ECO, 2008a: 349-350)

Questa premessa gli ha imposto una sequenza un-passo-avanti-e-tre-indietro, uno-avanti-e-due-indietro, uno-avanti-e-uno-indietro. E così, nel momento in cui 
appare la persona di padre Caspar, la trama si blocca per un po' nel momento presente della narrazione.

Accanto alla storia raccontata ci sono alcuni flashback attraverso i quali si viene a sapere ciò che è successo fino al momento in cui il romanzo ha inizio. In Sei passeggiate nei boschi narrativi si legge che in una narrazione nel tempo 1 (tempo in cui gli avvenimenti raccontati hanno luogo) sia il narratore (in prima o terza persona) che i protagonisti possono parlare di qualcosa che è accaduto prima degli avvenimenti raccontati. Come dice Gérard Genette, il flashback sembra recuperare ciò che l'autore ha dimenticato (ECO, 2007: 39). Umberto Eco non ha dimenticato niente: l'ha fatto apposta. E il pretesto per i flashback sono le metafore.

Ne L'isola del giorno prima abbiamo a che fare con una lingua barocca che vuole fare della metafora uno strumento di nuove conoscenze, un modo per cogliere somiglianze impreviste e per istituire nessi tra cose dissimili (FORCHETTI, 2005: 49). Il gusto secentesco della metafora permette a Umberto Eco di creare dei collegamenti tra elementi molto distanti fra loro: perciò Roberto - a causa dei vari impulsi o delle varie associazioni - rammenta diverse vicende della propria vita. Come dice il narratore del libro:

"Roberto lascia capire assai poco dei suoi sedici anni di vita prima di quella estate del 1630. Cita episodi del passato solo quando gli paiono esibire qualche connessione con il suo presente sulla Daphne [...]" (IP: 22).

Quindi ogni ricordo del protagonista è evocato da qualcosa che porta i segni della similitudine con ciò che accade o con ciò che egli vede, sente e così via durante il suo soggiorno sulla nave. Si vede che anche nel suo terzo romanzo Umberto Eco racconta la storia nel modo tipico per il suo stile narrativo, cioè "facendo un passo indietro" (ECO, 2008a: 324).

Il primo flashback di Roberto, ad esempio, avviene allorché sente cantare gli uccelli dell'isola che gli ricordano il fragore delle armi e dell'esercito schierato a battaglia nei pressi di Casale. Le grida di pappagalli, il canto di usignolo, merlo, calandra, rondini, il rumore delle cicale e dei grilli lo fanno pensare al rumore dei carriaggi, al cozzare delle armi, alle voci dei castigliani, al grugnito dei lanzichenecchi, a qualche suono di tromba ecc. insomma, gli fanno compiere delle associazioni assai lontane, visto che i suoni della natura non hanno niente a che fare con i suoni della battaglia e dell'esercito, però dall'altra parte sulla nave Roberto per la prima volta in vita sua sente davvero il canto degli uccelli e lo collega ai suoni che fino ad allora erano per lui i più naturali. Dunque il primo ricordo è stato evocato dall'udito.

Un altro flashback per esempio ha luogo quando Roberto si accorge di non essere solo sulla nave, che oltre a lui c'è anche qualcun altro, un "ospite nascosto", un intruso: "Ma, tormentandosi come si tormenta, Roberto non diventa fonte di pietra, e subito riporta l'angoscia che avverte all'altra angoscia provata a Casale [...]" (IP: 66). Questo flashback è stato evocato non da una sensazione fisica ma da un'emozione. Roberto, spaventato, pensa subito ad una situazione analoga, quando aveva provato lo stesso timore. Dopo questo ricordo si rende conto di rammentare per caso anche altre situazioni: 
“Arrestando per un attimo l'onda dei ricordi, Roberto si era accorto che aveva rievocato la morte del padre non per il proposito pietoso [...], ma per il mero accidente, mentre rievocava lo spettro di Ferrante, evocato dallo spettro dell'Intruso della Daphne" (IP: 74).

La catena dei ricordi non può essere del tutto controllata; alcune situazioni sono evocate perché "appartengono" ad un certo tempo, perché sono collegate ad altre situazioni in maniera inseparabile.

Non tutti i flashback sono provocati per similitudini tra cose lontane. Alcuni ricordi sono evocati dalle cose vicine, come per esempio quando gli orologi sulla Daphne gli fanno pensare al segreto del punto fijo. Il gran numero di orologi trovati sulla nave "in rotta su mari in cui il mattino e la sera sono definiti dal corso del sole" non può essere casuale. E il parallelo tra la situazione presente di Roberto e quella sull'altra nave, l'Amarilli, è ovvio. E perciò la sua memoria torna a quei fatti.

Guardando le pietre, Roberto si mette a riflettere sul concetto di memoria. Giunge alla conclusione che è una potenza dell' anima; che aver memoria significa aver nozione del prima e del dopo, perché altrimenti si crederebbe che le emozioni di cui ci si ricorda siano presenti nell'istante stesso in cui vengono ricordate; invece si sa che sono percezioni passate perché sono più deboli di quelle presenti. Dunque da quelle riflessioni risulta che non solo si ricorda attraverso le percezioni, ma anche che la memoria è strettamente collegata al tempo, e per ricordare bisogna avere il sentimento del tempo.

La memoria di Roberto - quella memoria stimolata dalle numerose somiglianze - diventa presenza insopportabile del passato che non può essere dimenticato. Roberto comincia a desiderare di liberarsi dai ricordi che gli giungono in abbondanza, e non sempre voluti, alla mente. Perciò inizia a immaginare che al centro dell'isola "si ergeva, invitante nei suoi colori tenui, l'Albero dell'Oblio, mangiando i cui frutti [...] avrebbe potuto trovare la pace. Smemorare. Trascorse così la giornata, neghittoso all' apparenza, attivissimo nello sforzo di diventare tabula rasa" (IP: 99). Tentativi vani perché - citando il narratore - "come accade a chi si imponga di scordare, più sforzi faceva, più la sua memoria s'animava". Un procedimento opposto si vedrà nel quinto romanzo di Umberto Eco, La misteriosa fiamma della regina Loana, nel quale il protagonista tenterà di uscire dalle oscurità dell'oblio, desiderando qualche "droga" per ricordare.

Come si può leggere nel libro, i ricordi di Roberto riguardanti le vicende accadute a Casale non sono provocati solamente dalle somiglianze con le vicende capitate durante il suo soggiorno sulla Daphne ma anche dalle differenze tra di loro:

"Perché Roberto rievoca Casale per descrivere i suoi primi giorni sulla nave ? Certo, c'è il gusto della similitudine, assediato una volta e assediato l'altra, ma a un uomo del suo secolo chiederemmo qualcosa di meglio. Caso mai della similarità dovevano affascinarlo le differenze, feconde di elaborate antitesi: a Casale era entrato di sua scelta [...] e sulla Daphne era stato gettato, e anelava solo a uscirne. Ma direi piuttosto che, mentre viveva una storia di penombre, riandava a una vicenda di azioni convulse vissute in pieno sole, in modo che le rutilanti giornate dell'assedio, che la memoria gli restituiva, lo compensassero di quel suo pallido vagabondare." (IP: 44) 
Anche se le differenze possono stimolare la memoria di Roberto, tuttavia più feconde e potenti, e perciò più significative, sono le similitudini. Non è diverso nel caso di Casale: per quanto riguarda l'assedio casalese, molti fatti di quel periodo assomigliano all'assedio oceanico che Roberto vive sulla nave: "Si viveva a Casale, nella Cittadella, come sulla Daphne: immaginando un'Isola lontana, e con gli intrusi in casa" (IP: 134). Roberto torna a quei giorni con la memoria non per puro caso. Come dice il narratore:

"E non mi pare un semplice accidente della memoria. Ho già detto che Roberto mi sembra far collidere quegli eventi lontani e la sua esperienza sulla Daphne come per trovare dei nessi, delle ragioni, dei segni del destino. Ora direi che il riandare ai giorni di Casale gli serve, sulla nave, a rintracciare le fasi per cui, giovinetto, stava lentamente apprendendo che il mondo si articolava per stranite architetture." (IP: 50)

Non è un caso che il frammento citato si trovi all'inizio del capitolo intitolato Il Labirinto del Mondo. È un tema che era già presente ne Il nome della rosa. L'isola del giorno prima presenta dei tratti caratteristici del romanzo di formazione; il giovane protagonista inizia a conoscere il mondo. Così come è labirintica la nave sulla quale si trova, così è labirintico il mondo nel quale Roberto deve imparare i segreti del secolo in cui vive. Non è facile: questo mondo è molto complicato, rizomatico, e perciò infinito. Già a Casale Roberto ha capito che il mondo è un grande mistero, complicato e impossibile da risolvere. Il narratore confessa che:

"Dal modo in cui ne rievoca sulla Daphne ritengo che a Casale, mentre perdeva padre e se stesso in una guerra dei troppi e dal nessun significato, Roberto avesse appreso a veder l'universo mondo come un insicuro ordito di enigmi, dietro al quale non stava più un Autore; $\mathrm{o}$, se c'era, pareva perduto nel rifar se stesso da troppe prospettive." (IP: 137)

Quella rete di enigmi, dai quali è composto 1"'universo mondo", sembra essere fuori il controllo persino del suo Autore perché nessuno è capace di regnare su quella molteplicità labirintica delle connessioni tra le cose. Capire il mondo è molto difficile; però padre Emanuele dà a Roberto l'indicazione su che cosa sarebbe necessario per poterlo fare, dicendo che:

"[...] per capire la Cosa Pensante, ovvero il nostro modo di conoscere il Mondo, noi non possiamo che usare un altro Cannocchiale, [...] e che non è né tubo né lente, ma Trama di Parole, Idea Perspicace, perché è solo il dono dell'Artificiosa Eloquentia quel che ci consente di capire questo Universo." (IP: 84)

Però il labirinto che domina nel libro è quello della nave, di "questo carcere inespugnabile". Pur avendo passato molti giorni sulla nave, Roberto si rende conto che la Daphne "poteva celare ancora tali e tante sorprese, che egli avrebbe potuto trascorrere la vita a cercare di violarla, senza speranza?". E seguendo lo spirito del secolo ha definito la nave come fonte di "Divise Enigmatiche" e ne ha apprezzato la metafora.

La ricchezza allusiva della Daphne non consiste solo nel fatto che fa rammentare le vicende del passato di Roberto. Essa diventa anche per lui emblema del corpo della donna amata. Come racconta il narratore: 
"Un giorno aveva identificato Daphne, la nave, con il corpo della amata. Non era forse Daphne una creatura che si era trasformata in lauro - in sostanza arborea, dunque, affine a quella da cui la nave era stata tratta? La melodia gli cantava dunque di Lilia” (IP: 318).

La melodia qui menzionata è un gioco di coincidenza: la nave ha nome Daphne, che è anche il titolo di una delle più note composizioni musicali di Jacob van Eyck, compositore del Seicento olandese, Does Daphen d'over schoone Maeght, con la quale Roberto viene a contatto prima in Olanda, e che risente una seconda volta sulla nave, suonata dall'organo ad acqua di padre Caspar. Come relaziona il narratore, lo stesso Roberto si dice subito che è ingegnosissimo emblema udire una musica per flauto detta "Daphne" ed essere su di un fluyt (un 'flauto') chiamato Daphne. ${ }^{3}$ Questo fatto è un altro aspetto allusivo della navelabirinto.

La memoria è molto presente nei romanzi di Umberto Eco. F. Forchetti mette in rilievo il fatto che la narrativa echiana gravita intorno ai giochi e ai paradossi della memoria (FORCHETTI, 2005: 273). Infatti, ogni romanzo ne dà la prova. Ne L'isola del giorno prima il labirinto della nave, la Daphne, diventa - secondo lo spirito del tempo in cui si svolge la storia - il vero Teatro della Memoria. Questo teatro mnemonico ossessiona Roberto con suggestioni e somiglianze. E la memoria di Roberto è un ricordare in barocco che nasce dalle sollecitazioni sensoriali e dal gusto della metafora. Nel romanzo ambientato nel Seicento, la metafora è assolutamente indispensabile nella ricerca delle somiglianze, delle analogie e delle corrispondenze tra le cose. Non senza importanza è l'allusione nella persona di padre Emanuele - a uno dei più conosciuti teorici della letteratura, Emanuele Tesauro, autore del trattato sulla metafora e sul concetto Il cannocchiale aristotelico. Il fascino barocco delle somiglianze si manifesta nel libro nel produrre delle metafore inaspettate; e quelle metafore hanno un ruolo molto importante, in quanto sono pretesto per i ricordi del protagonista.

\section{BIBLIOGRAFIA}

BolzONi Lina (1991), Lo spettacolo della memoria, introduzione a G. Camillo, L'idea del Theatro, Palermo, Sellerio.

BondANELla Peter (1997), Umberto Eco. Semiotyka, literatura, kultura masowa, Kraków, Znak. (titolo originale: Umberto Eco and the open text. Semiotics, fiction, popular culture)

ECO Umberto (2005), L'isola del giorno prima, Milano, Bompiani.

Eco Umberto (2007), Sześć przechadzek po lesie fikcji, Kraków, Znak. (titolo originale: Six Walks in the Fictional Woods)

ECO Umberto (2008a), Come scrivo, in Sulla letteratura, Milano, Bompiani.

ECO Umberto (2008b), La "Poetica" e noi, in: Sulla letteratura, Milano, Bompiani.

ECO Umberto (2009), Od drzewa do labiryntu, in: Od drzewa do labiryntu. Studia

\footnotetext{
3 “'....] paragonando con 1'Amarilli, Roberto ne concluse che anche la Daphne era un fluyt olandese, o flauto, o flûte, o fluste, o flyboat, o fliebote, come variamente si chiamavano quelle navi da commercio e di media stazza [...]." (IP : 11)
} 
historyczne o znaku i Interpretacji, Warszawa, Aletheia. (titolo originale: Dall'albero al labirinto. Studi storici sul segno e l'interpretazione)

FORCHETTI Franco (2005), Il segno e la rosa. I segreti della narrativa di Umberto Eco, Roma, Castelvecchi.

SAMBo Marco M. (2004), Labirinti. Da Cnosso ai videogames, Roma, Castelvecchi.

YATES Frances A. (1977), Sztuka pamięci, Warszawa, Państwowy Instytut Wydawniczy. (titolo originale: The Art of Memory). 